\section{Hang over}

Helga Peter ${ }^{1}$ und Thomas Penzel ${ }^{2}$

${ }^{1}$ Marburg, Deutschland

${ }^{2}$ Interdisziplinäres Schlafmedizinisches Zentrum, Charité -

Universitätsmedizin Berlin, Berlin, Deutschland

\section{Definition}

Nachwirkungen einer Medikation vom Vortag. Häufig relevant bei der Verabreichung von langwirkenden $>$ „Hypnotika“. Siehe auch $\triangleright$ „Benzodiazepine“. 